Cahiers de la recherche sur les droits

Cahiers

$\mathrm{F}^{\text {sur les }}$ Droits fondamentaux

$2 \mid 2003$

Les titulaires particuliers des droits fondamentaux

\title{
Religions, sectes et discriminations
}

Philippe Gast

\section{OpenEdition}

Journals

Édition électronique

URL : https://journals.openedition.org/crdf/7707

DOI : $10.4000 /$ crdf. 7707

ISSN : 2264-1246

Éditeur

Presses universitaires de Caen

Édition imprimée

Date de publication : 1 janvier 2003

Pagination : 49-62

ISBN : 2-84133-205-5

ISSN : 1634-8842

Référence électronique

Philippe Gast, « Religions, sectes et discriminations », Cahiers de la recherche sur les droits

fondamentaux [En ligne], 2 | 2003, mis en ligne le 18 décembre 2020, consulté le 15 novembre 2022

URL : http://journals.openedition.org/crdf/7707 ; DOI : https://doi.org/10.4000/crdf.7707 


\title{
Religions, sectes et discriminations
}

\author{
Philippe GAST \\ Maître de conférences \\ Faculté des Affaires internationales du Havre
}

I. La dangerosité du dogmatisme religieux

A. Laïcité et religions traditionnelles

B. Les nouvelles religions et la laïcité

II. Plaidoyer pour une investigation sérieuse sur les mouvements religieux

A. Le fondement conceptuel de la méthode de travail d'un Observatoire objectif

B. Le fonctionnement d'un Observatoire

Confucius disait que lorsque le sens des mots disparaît c'est la liberté qui se perd... Commençons donc par définir quelques-uns des termes de notre sujet avant d'en envisager les problématiques et les tentatives de solution.

La caractéristique essentielle de la religion ${ }^{1}$ est de proposer une métaphysique exposant une forme ou une autre d'immortalité, de non-mort d'une partie de la conscience. Plus encore (et contrairement aux philosophies qui peuvent aussi proposer ce genre d'hypothèses) la religion propose d'expérimenter cet état intemporel, à l'exemple de son fondateur. La religion est d'abord et avant tout née de l'expérience spirituelle de son fondateur, qui a décrit une expérience subjective de l'éternité, d'un état intemporel perçu comme transcendant la mort corporelle.
Historiquement, la plus ancienne manifestation religieuse semble être apparue avec l'homme du Néanderthal $^{2}$ qui créa le premier temple et qui fut le premier hominidé à enterrer ses morts avec les premières traces d'une croyance à une conscience post mortem. C'est le premier à avoir eu l'intuition de la question : ne suis-je qu'un corps mortel ou une âme immortelle?

L'homo sapiens, notre ancêtre direct, fut le disciple du Néanderthal avec lequel il partagea quelques milliers d'années d'histoire, et fut initié par lui à cette quête de l'identité, mais son maître ne lui survécut pas. En effet il est probable que pour l'homo sapiens, sa capacité de langage, liée à la facilité d'élocution découlant d'une morphologie de la mâchoire, et une langue plus fine que le Néanderthalien lui permirent de vocaliser plus de sons et

1. À partir de l'étymologie religare, relier on trouve en fait trois liens: le lien collectif, objectif ou «horizontal», le lien subjectif, spirituel ou vertical de contact entre des états de conscience «normaux» et des états modifiés (intemporels en particulier) et le lien dogmatique qui consiste à affirmer que les paroles du fondateur sont incontestables: elles sont La Vérité.

2. Premier hominidé à habitat structuré (entre - 300000 et - 40000 ans) mais qui semble avoir coexisté pendant plusieurs milliers d'années avec les homo sapiens, puisqu'on a trouvé en Galilée des silex d'homo sapiens datés par thermoluminescence de - 90 ooo ans, soit plusieurs milliers d'années avant les silex néanderthaliens datés en Europe de - 60000 ans. L'homme du Néanderthal est le premier à avoir enterré ses morts avec des traces de croyances en l'immortalité d'une âme (offrandes, nourriture post mortem), c'est donc le premier « animal » à avoir conçu que la vraie nature humaine ne se réduit pas au corps, marque d'une première quête d'identité qui sera celle de Descartes bien plus tard (le «je suis », derrière le « je pense » de son Discours de la méthode, repris par Kant dans la notion d'aperception transcendantale dans sa Critique de la raison pure, puis par Husserl dans son ouvrage Idées directrices pour une phénoménologie à travers les concepts de Noèse (côté sujet de la conscience) et la noème (coté objet de la conscience). 
donc de nommer plus de choses, et par conséquent de maîtriser plus d'informations et de concepts pour finalement devenir sapiens-sapiens... et survivre à la sélection des espèces.

C'est la première dimension de la religion ${ }^{3}$, la dimension intérieure, subjective, la recherche d'états modifiés de conscience, d'un état intemporel : c'est le lien vertical, celui qui relie les états de la conscience vers l'identité essentielle, spirituelle ${ }^{4}$. En effet ce «je suis» est précisément la définition de Dieu dans les religions bibliques (eyeh asher eyeh-Exode 3,12 ) ou hindoue ( aham so ham ${ }^{5}$ ) ou encore musulmane soufie (anal $\left.\mathrm{Haq}^{6}\right)$. L'expérience spirituelle est donc l'expérience qu'un individu peut faire du «je suis $»^{7}$ derrière le «je pense ». C'est l'essence de l'individu, ce qui reste dans sa conscience, quand il a néantisé (pour reprendre la terminologie des mystiques catholiques rhénans médiévaux, du Bouddhisme ou de l'Hindouisme védantique) ou quand il a plongé dans la Nuit Obscure (pour reprendre la terminologie de saint Jean de la Croix ou celle de Moïse) les perceptions sensorielles, émotives et mentales, et qu'il ne reste plus que la conscience pure, la conscience qui observe la conscience, expérience que le langage ne peut décrire car le sujet a fusionné avec l'objet (et une phrase ne peut exister sans sujet distinct d'un objet). La terminologie en est d'ailleurs parfois identique: ainsi l'État divin décrit par Moïse comme "je suis qui je suis" (eieh asher eieh - Exode, 3, 12), où grammaticalement le sujet est fusionné avec l'objet, se retrouve à l'identique dans la formule brahmanique des Upanisad: "hamsa so'ham», "je (suis) cela, cela je (le suis) »... mais l'idée est la même lorsque le Christ annonce: «le Père est en moi comme je suis dans le Père» (Jean, 1,38 ) ou "le royaume des cieux est en vous » (Luc, 17, 21); ou encore quand Al Hallaj annonce «ana’l Haqq», «je suis Dieu» (dans son aspect vérité).

Les problèmes, en matière de religion, apparaissent quand les successeurs n'ayant pas l'expérience intérieure, verticale, en ont une vision mentale par la mémorisation de la description faite par le fondateur. Alors naît la dimension qui progressivement devient dogmatique car éloignée d'une expérience vécue.

Or il est clair que chacun peut proposer une hypothèse d'immortalité pour répondre à cette peur fondamentale de ne plus être. Il s'agit là d'une liberté fondamentale, saine en démocratie et inévitable depuis que l'homme est Néanderthal. Mais les hypothèses de non-mort étant évidemment différentes ${ }^{8}$, les conflits métaphysiques sont d'autant plus passionnés qu'ils réveillent la peur de la mort enfouie au plus profond de chacun. Si l'on rajoute à cette peur fondamentale les intérêts que tel ou tel groupement religieux ${ }^{9}$ a à conserver sa mainmise sur la société (quand ce ne sont pas de vils intérêts financiers ou une simple volonté de puissance - déformation du lien horizontal), on comprend mieux que face à l'émergence de nouveaux mouvements religieux concurrents, dès que l'on aborde ces questions, l'on soulève des conflits d'une violence anachronique dans un monde prétendu laïque et scientifique!

En effet on constate que le fonctionnement des religions historiques une fois installées a souvent dérivé vers le dogmatisme où l'on observe qu'une minorité cléricale impose alors à la majorité son interprétation du texte fondateur, en refusant tout compromis. Ainsi par exemple c'est vers le XIV ${ }^{\mathrm{e}}$ siècle que l'Islam a figé sa jurisprudence quant à l'interprétation du Coran, considérant que tous les problèmes ayant été traités, il n'y avait plus lieu à de nouvelles interprétations.

Ce comportement, soulignons-le, est incompatible avec les principes démocratiques de liberté de critique car la science comme la démocratie respectent la loi majoritaire, et la critique minoritaire. La première fondant la stabilité du système et la seconde sa dynamique.

La religion est donc la synthèse de trois liens : social, intérieur - relatif à l'éternité - et dogmatique (les paroles du fondateur sont LA VÉRITÉ).

La discrimination consiste à instaurer une inégalité entre les êtres. En violant le principe d'égalité elle s'oppose au principe démocratique ${ }^{10}$. Ceci impose que le

3. Dont on peut rappeler que l'étymologie re-ligare donne le mot «re-lier», «lien » (une autre possible fait référence à legere, lire, référence au texte fondateur).

4. Le « je suis » derrière le « je pense » de Descartes. En effet, le mot «spirituel », dans son étymologie, provient de spiritus, l'essence, le principe, ce qui est premier; Descartes en a eu une magistrale intuition dans ses Méditations métaphysiques et surtout dans son Discours de la méthode. Constatant que l'on peut douter de tout: des sens, des idées... une chose néanmoins demeure stable, c'est que «je pense donc je suis", constatation qu'il complète plus bas dans le même texte par l'expression «pour penser il faut être». La spiritualité est donc cet état de conscience qui est premier sur toute autre perception. C'est le «je suis» derrière le « je pense». L'état «je suis» au-delà même de la pensée « je suis ». Cette expérience, seule certitude que peut avoir l'humain, permit à Descartes de conclure que je ne peux être sûr que de deux choses: mon existence et celle de Dieu (qui se définit comme «Je suis »).

5. De Yajnavalkya dans la Brhadàranyakaupanisad. Ces deux dernières citations faisant apparaître clairement et de façon identique une structure grammaticale où l'objet et le sujet sont confondus, état de non-dualité incontestable.

6. Mansur Al Hallaj, dans son Diwan.

7. Non pas, « je suis» quelqu'un, avec tel passé, tel avenir, telle ou telle étiquette, ce qui définit l'ego et fonde ses boursouflures, mais état «je suis » sans objet qui est un état de conscience pure, non dualiste, dont l'humilité est la caractéristique psychologique universelle. Cette caractéristique est très représentative de la différence entre des escrocs sectaires ou ecclésiastiques qui recherchent le pouvoir sur autrui et les vrais mystiques philanthropes et désintéressés.

8. Immortalité «personnelle» dans les religions bibliques ou dans les versions hindoues ou bouddhistes de la réincarnation, immortalité impersonnelle dans les courants nihilistes (certaines approches nirvaniques) ou immortalité à la fois personnelle et impersonnelle dans les courants non dualistes.

9. Traditionnels ou athées (marxistes en particulier, ainsi contrairement à la décision du Conseil d'État du 17 juin 1988, la Commission européenne des droits de l'homme a justement affirmé que l'athéisme est une forme de religion car affirmer que Dieu existe ou qu'il n'existe pas, c'est aussi une forme de dogme métaphysique... Cf. Rapport Guyard, p. 88).

10. Démos-cratos : le pouvoir du peuple selon la formule - fort peu mise en pratique - de la Constitution de la Veépublique : « république du peuple, par le peuple, pour le peuple.» 
but de la démocratie soit de satisfaire tout le peuple. Le but du droit (et de la société) dans cette perspective est par conséquent de satisfaire tous les êtres sur tous les plans: matériel, intellectuel, spirituel. Mais si satisfaire tout le peuple consiste en priorité à satisfaire la majorité, c'est aussi satisfaire les minorités ${ }^{11}$.

Ce respect des personnes impliquant nécessairement la reconnaissance de leur autonomie de volonté et de leur liberté fondamentale ${ }^{12}$ quant à leur choix de vie aboutit à la constitution d'un droit subjectif majeur qui est la non-ingérence de l'État dans leur vie privée à travers les mœurs et la morale qui sont hors du champ d'application du droit ${ }^{13}$. Nul ne sait mieux que quiconque ce qui est bon pour lui, que lui-même. La séparation de la sphère privée et publique est donc aussi indispensable à la démocratie que le respect de la majorité, au risque d'aboutir à la dictature de la majorité que dénonçait Tocqueville au XIX ${ }^{\mathrm{e}}$ siècle $^{14}$. Ces fondements de la démocratie sont aussi d'ailleurs ceux du mécanisme d'élaboration des sciences (respect de la loi majoritaire et respect de la critique minoritaire). Les deux notions sont intimement liées.

D’une part, historiquement, elle sont nées ensemble dans l'Antiquité grecque d'abord, puis ont resurgi en même temps au Siècle des Lumières en Europe de l'Ouest et en France en particulier; d'autre part (et ce n'est pas un hasard) elles sont la manifestation d'un même fonctionnement: le respect du consensus majoritaire et le respect de la critique minoritaire.

En effet : d'une part, pour qu'une loi soit démocratique, il faut qu'elle représente la volonté générale (chère à Rousseau), représentée par la majorité des citoyens; mais cette loi peut être remise en cause à tout moment, et dès que les critiques minoritaires deviennent majoritaires, on la modifie; d'autre part, pour qu'une loi soit scientifique, c'est-à-dire déclarée objective, juste, il faut qu'elle recueille l'assentiment de la majorité de la communauté scientifique; néanmoins elle peut être remise en cause à tout moment par une expérience qui lui serait contraire (c'est la pratique du doute systématique de Descartes). On a donc bien les mêmes mécanismes qui prévalent dans les deux systèmes.

Ces deux systèmes avec leur méthode commune ont fait la force de la civilisation occidentale car le respect du consensus majoritaire assure une certaine stabilité, une certaine paix sociale; alors que le respect de la critique minoritaire assure la capacité d'évolution et d'adaptation du système. Que l'Occident la perde (par la pensée unique) et il perdra sa primauté.
Deux questions apparaissent alors : quels liens doitil y avoir entre droit et religion et comment concilier Religion et Démocratie, puisque les critères sont particulièrement inversés? À la première question deux solutions sont apparues dans l'histoire, celle de la religion d'État, qui a été le système adopté par les monarchies de droit divin (Égypte, Chine, Japon, Rome, France...), et celle de la laïcité dans les systèmes démocratiques.

La deuxième question fait encore problème aujourd'hui. La laïcité est le mécanisme qui dans une démocratie permet la mise en œuvre de la non-discrimination. En effet un État laïc est un État non religieux : un laïc est celui qui n'est pas clerc, qui n'appartient pas à une église. Le concept est apparu la première fois dans l'histoire européenne avec Galérius, empereur romain qui après avoir persécuté les chrétiens décida de l'édit de Tempérance, au $\mathrm{III}^{\mathrm{e}}$ siècle, grâce auquel on pouvait être citoyen romain, et adhérer à une religion différente. Galérius fut suivi par Constantin qui déclara le Christianisme religion d'Empire, puis par Théodose qui imposa cette religion comme la seule de l'Empire: c'est lui qui détruisit tous les temples grecs (Delphes...) et en échange, nomma les évêques... Ce qui signifie qu'à partir du $\mathrm{IV}^{\mathrm{e}}$ siècle les évêques n'étaient plus des spirituels mais des politiques...

Puis on trouve Henry IV avec son édit de Nantes, puis l'édit de Tolérance de Louis XVI et enfin la proclamation de la liberté de religion de la Déclaration des droits de l'homme de 1789.

La laïcité apparaît comme une solution, voire comme un corollaire nécessaire de la démocratie car elle laisse la liberté à chacun et à chaque groupe de pratiquer, croire (c'est la dimension personnelle, privée, verticale), échanger, s'exprimer (dimension publique, horizontale). Au contraire la monarchie (dans son sens étymologique) s'accommode mieux de la religion unique car elle ne supporte pas la contestation.

Mais deux formes de laïcité sont possibles: celle, inclusive, en vigueur en Allemagne (système du concordat où l'État rémunère les religieux - mais il faut alors reconnaître toutes les religions si l'on ne veut pas qu'il y ait de discrimination); l'autre exclusive, en vigueur en France (par laquelle l'État ne reconnaît ni ne subventionne aucun culte - article 2 de la loi de 1905 - mais qui reste un beau principe tout de suite contredit par l'article 19 qui autorise les déductions fiscales aux associations cultuelles, ce qui donc oblige l'État à dire quelle association est cultuelle ou pas et donc... reconnaît que telle association est un culte et telle autre pas, faisant apparaître du coup l'arbitraire et l'irrationnel.

11. Que représente d'ailleurs chaque individu car chaque individu étant un unique agglomérat de désirs, de peurs, de mémoires émotionnelles, il est singulier et donc nécessairement minoritaire; imposer une morale même majoritaire aboutirait à terme à un étouffement des libertés.

12. Définie remarquablement dans l'article 4 de la Déclaration des droits de l'homme de 1789 : «La liberté consiste à pouvoir faire tout ce qui ne nuit pas à autrui. »

13. On rappellera que le mot latin mores a donné les mots français «mœurs» (mode de vie) et «morale» (principes des actes de la vie). Saint Thomas d'Aquin puis Kant ont fait la distinction fondamentale entre la morale (règles qui sont celles de la vie privée, et qui sont donc facultatives) et le droit (qui sont les règles collectives et obligatoires). La distinction antique tentée par Aristote entre Dikaios (l'homme qui agit selon des règles de vie justes) et to Dikaion (Justice, au neutre) n'est pas pertinente car la règle de vie concerne aussi bien la vie privée que publique, la morale et le droit comme la notion d'action juste. Il y a donc un risque de confusion. La séparation entre la morale comme règle de vie privée et facultative d'un côté, et du droit comme règle collective et obligatoire, au contraire est extrêmement pertinente dans la perspective démocratique et libérale.

14. Dans La Démocratie en Amérique. 
Comme le rappelle le rapport parlementaire sur le financement des sectes du 10 juin $1999^{15}$, aucune nouvelle religion n'a été reconnue en France: est-ce normal en application du principe de non-discrimination laïque? Et tout d'abord qu'est-ce qu'une secte? Le rapport parlementaire de 1995 a considéré qu'il n'était pas possible de définir la notion... et pourtant propose dix critères qui caractériseraient une secte (sic). Outre le caractère plus que fantaisiste des critères ${ }^{16}$ il y a une contradiction manifeste dans cette approche!

Si l'on essaye d'y voir plus clair sur la notion de secte, il faut se référer à l'étymologie qui ramène à l'idée de se couper d'une structure, ou d'une doctrine existante (secare) en suivant une nouvelle doctrine ou une nouvelle structure ou un nouveau maître (sequi). On constate d'ailleurs que toutes les religions ont été des sectes au sens étymologique, à leur origine: le Judaïsme s'est fondé par le fait pour les premiers juifs de suivre Abraham qui a quitté la ville mésopotamienne d'Ur (fuite ou expulsion?) dont il rejetait les dieux en prônant l'adoration d'un Dieu unique; les chrétiens se sont coupés des juifs en suivant l'enseignement du Christ (puis les catholiques se sont coupés des orthodoxes, les protestants des catholiques, etc.), les musulmans se sont coupés des juifs et des chrétiens en suivant la doctrine de Muhammad (puis les chiites se sont coupés des sunnites et les soufis des deux autres).

En Inde, les bouddhistes se sont coupés de la doctrine brahmanique en rejetant les rituels et les castes pour suivre la doctrine du Bouddha dans la recherche de l'éveil personnel et intérieur (dans le Bouddhisme les mahayanistes se sont coupés des hinayanistes, et les vajrayanistes, des mahayanistes...).

Mais le mot secte est devenu à partir du $\mathrm{XVI}^{\mathrm{e}}$ siècle un terme négatif utilisé par les catholiques pour stigmatiser les protestants considérés comme hérétiques. La connotation négative est donc restée dans le langage actuel, sauf que dans un État laïc, il ne saurait sans discrimination conserver la même acception. Il faut donc réactualiser son sens. Or précisément dans un État de droit, démocratique, libéral et laïc, le seul sens négatif que l'on peut associer à une structure est le fait qu'une structure viole l'État de droit par la mise en œuvre de sa doctrine.

On retrouve donc sur le fond les trois « liens » dans la secte comme dans la religion. La différence réside dans le fait de commettre des actes illégaux en rapport avec sa doctrine. Pourtant la guerre menée par certains contre les sectes ne se fonde pas sur ce critère.

Pourquoi ce refus et cette discrimination? La dangerosité des sectes? Par rapport à quoi? N'est-ce pas une guerre idéologique dissimulée? Une nouvelle forme de guerre de religion ${ }^{17}$ ?
Mais les anciennes religions ne sont-elles pas aussi (voire plus) dangereuses eu égard à l'origine de la plupart des guerres et des violences d'aujourd'hui? Les guerres aujourd'hui ne sont-elles pas toutes religieuses (même si c'est souvent un prétexte) ? Catholiques / protestants en Irlande, juifs / musulmans en Israël, musulmans / hindous entre Pakistan et Inde, chrétiens / musulmans en Indonésie et aux Philippines, au Soudan, en Érythrée, à Chypre, hindous / bouddhistes à Ceylan... Le terrorisme en dehors des guerres ouvertes apparaît partout. L'arbre des sectes ne cacherait-il pas la forêt des religions fanatiques établies?

La dangerosité religieuse est à considérer en bloc (I) à cause de son dogmatisme qui s'oppose au fondement de la démocratie en devenant fanatisme, qu’il soit religieux ou sectaire (au sens étymologique du terme); mais comment la démocratie peut-elle s'en prémunir sans discrimination ni atteinte à la liberté de culte (liberté publique) et de croyance (liberté de la vie privée) autrement que sur la base d'une recherche objective et bien fondée conceptuellement (II)?

\section{La dangerosité du dogmatisme religieux}

Le monde politico-médiatique utilisant souvent la distinction religion / secte, il convient de distinguer les anciennes religions (A) des nouvelles (B) afin de réaliser que les unes et les autres peuvent être pacifiques ou au contraire nuisibles à l'ordre public et à la paix sociale.

\section{A. Laïcité et religions traditionnelles}

Les religions bibliques d'un côté, qui ont développé une vision causale de la divinité, l'aspect créateur étant mis en avant, qui aboutit à une séparation, une dualité Dieu / Création, d'où découle les dualités Dieu / Diable et Bien / Mal, et la suite logique qui aboutit à ce que les «bons» doivent détruire les «méchants ». Cette conceptualisation de base génère immanquablement la violence et l'intolérance, ce qui se vérifie par l'histoire de ces religions qui ont été conquérantes par les armes (épisode du Veau d'or dans le Judaïsme et des différentes guerres liées à la «terre promise », Inquisition chez les chrétiens, Djihad chez les musulmans).

Les religions védiques (Hindouisme, Bouddhisme) d'un autre coté ont développé une approche globalisante de la divinité qui est présentée comme tout englobante (le bien et le mal y sont alors des notions relatives à chaque être et inhérentes à l'Être, voire la manifestation d'une ambivalence paradoxale de l'Être-non Être (la

15. Rapport $\mathrm{n}^{\circ} 1687$, «Les sectes et l'argent», p. 66

16. Rapport $\mathrm{n}^{\circ} 2468$, «Les sectes en France», dont j'ai fait une critique dans un colloque sur les libertés fondamentales à l'Université du Havre le 15 mai 1997, intervention publiée dans un article dans l'ouvrage collectif Les Droits fondamentaux de la personne humaine en 1995 et 1996 (Paris, L'Harmattan, 1998).

17. On constatera par exemple que les ennuis du Mandarom ont commencé par des articles négatifs issus du journal La Croix quelque temps après que le fondateur se soit proclamé messie « cosmoplanétaire»... 
«vacuité» chez les bouddhistes en particulier). On a donc une vision moins dualiste et conflictuelle, ces qualités étant par nature relatives à l'expérience personnelle et donc reléguées par nature à la vie privée. Dans cette approche on aura plutôt tendance à considérer les êtres en fonction de leur degré d' «évolution spirituelle» plutôt que comme porteurs d'un Bien ou d'un Mal absolus. Point besoin de violence ou de volonté de puissance, car chacun construit son propre bonheur ou son propre malheur comme résultat de ses pensées, paroles, actes (loi du karma).

De ces deux approches, la seconde paraît plus facilement adaptable à la démocratie et à la laïcité car le spirituel est affirmé comme étant de l'ordre de la vie privée.

Néanmoins dans certaines circonstances, les religions établies peuvent faire apparaître un trouble à l'ordre public (1) mais aussi peuvent être des facteurs de paix sociale (2).

\section{Le fanatisme dans les religions traditionnelles}

On distinguera les religions bibliques (a) des religions védiques (b).

\section{a. Le fanatisme dans les religions bibliques \\ $1^{\circ}$ Judaïsme}

Le Judaïsme a développé le principe de l'hérédité matrilinéaire (pour être juif il faut être de mère juive) vraisemblablement comme principe d'autoprotection pour éviter la dissolution, depuis la diaspora liée à la destruction du temple de Jérusalem par les Romains. Cette situation a eu une conséquence dangereuse et incompatible avec la démocratie.

En effet, la promotion et l'identification des juifs avec une certaine forme de supériorité (peuple élu de Dieu) héréditaire, constituent (comme le système de castes en Inde dans sa version rigoriste) une forme de racisme (classification des humains en catégories; hiérarchie de ces catégories en «supérieure » ou «inférieure» sur un plan psychologique, intellectuel ou spirituel - élu de Dieu ou pas; et critère héréditaire).

Néanmoins il faut constater d'une part que cette hérédité a pour effet l'absence de projet de conversion et par conséquent ne génère pas de violence autre que comme autodéfense ${ }^{18}$ ou en relation avec certains points de la doctrine comme celui de la «terre promise» qui génère les violences liées à la création de l'État d'Israël; et d'autre part qu'en outre, le statut de non-juif dans le Judaïsme ne semble pas avoir d'effet juridique vraiment discriminant. Certes la législation étant liée à la souveraineté, seul l'État d'Israël pourrait en être un exemple. Mais si l'Israël antique, pendant ses rares périodes d'indépen- dance, a connu l'esclavage et d'autres formes de discrimination (vis-à-vis des femmes), l'Israël contemporain ne connaît pas de discrimination juridique, ce qui est important... mais c'est juridiquement un État démocratique...

On notera qu'il n'y a pas dans le Judaïsme de vision conquérante contrairement au Christianisme et à l'Islam, il constitue simplement un "égoïsme collectif».

\section{$2^{\circ}$ Christianisme}

L'histoire de la conquête européenne par le Christianisme à partir de Théodose puis de Clovis s'est faite par la violence des armes, sauf en Irlande, où les druides se sont convertis, élaborant une sorte de religion de synthèse qui perdura jusqu'au XII ${ }^{\mathrm{e}}$ siècle où l'Angleterre envahissant l'Irlande avec la bénédiction de Rome y imposa des règles plus catholiques... Par ailleurs, on peut constater que l'Église catholique, qui aujourd'hui semble prôner la doctrine de la Déclaration des droits de l'homme, a été la première à la combattre au XVIII ${ }^{\mathrm{e}}$ siècle quand elle est née. On peut rappeler par exemple que le 10 mars 1791, le pape Pie VI condamne «cette liberté absolue qui non seulement assure le droit de n'être point inquiété sur ses opinions religieuses, mais qui accorde encore cette licence de penser, d'écrire, et même de faire imprimer impunément en matière de religion tout ce que peut suggérer l'imagination la plus déréglée: droit monstrueux [...]. Que pouvait-il y avoir de plus insensé que d'établir parmi les hommes cette égalité et cette liberté effrénée qui semble étouffer la raison? ${ }^{19}$. Cette condamnation était d'ailleurs la même que celle subie par la Magna Carta $^{20}$ de la part du pape Innocent III : « Nous rejetons et condamnons catégoriquement cette convention et interdisons sous peine d'excommunication tant au roi de l'appliquer qu'aux barons d'en réclamer l'application. Par la présente, nous déclarons cette charte nulle et dénuée de toute validité, sans révocation possible.»

On pourrait certes rétorquer que la doctrine politique de l'Église a évolué, étant donné de toutes façons que le Christ a toujours refusé de donner des règles politiques autres que de « rendre à César ce qui est à César et à Dieu ce qui est à Dieu », toute la doctrine politique de l'Église n'apparaissant alors que comme une excroissance, au gré des intérêts politiques.

Mais si l'on regarde le $\mathrm{XX}^{\mathrm{e}}$ siècle, on s'aperçoit que l'Église a pactisé avec le fascisme (Accords de Latran du 11 février 1929), avec le nazisme (Concordat du 20 juillet 1933), avec le franquisme par une allégeance des évêques au régime à travers une lettre collective en $1937^{21}$, soutien qui continua pendant tout le règne de Franco, alors même que Vatican II avait donné les orientations favorables aux droits de l'homme depuis les années soixante. Sans parler de la complaisance plus ou moins affichée vis-à-vis des

18. Néanmoins, et paradoxalement, en liant la pureté spirituelle à l'hérédité (le Peuple élu) on crée une forme de discrimination qui s'apparente à une forme de racisme... comme on en trouve également la marque dans la décadence brahmanique qui a généré le système de castes en Inde, qu'a vigoureusement rejeté le Bouddha.

19. Cité par C. Paturel, Sectes, religions et libertés publiques, Paris, La pensée universelle, 1996, p. 25, d'après A. Latreille, L'Église catholique et la Révolution française.

20. Première limitation des pouvoirs de la monarchie anglaise que lui imposèrent ses barons en 1215, et en cela ancêtre de la Déclaration française, en imposant le principe de la légalité des poursuites.

21. J. Comby, Pour lire l'histoire de l'Église, Paris, Cerf, ???. 
dictatures d'Amérique du Sud qui ressemblait parfois à un véritable soutien pendant les années soixante-dix. Aussi si l'on peut prendre acte de ce revirement de la politique de l'Église, il faut garder à l'esprit que ce peut n'être simplement que de l'opportunisme... Opportunisme d'autant plus choquant que le Christ demandait à ce qu'on prêche par l'exemple et que lui-même a préféré se faire tuer plutôt que de renier son message ${ }^{22}$ !

Cet opportunisme peut en effet à tout moment voir un revirement dans la doctrine politique de l'Église car c'est sa structure même qui est monarchique (le pape nomme les cardinaux qui nomment le pape, lequel a tous pouvoirs... en proclamant même le dogme de l'infaillibilité depuis le XIX ${ }^{\mathrm{e}}$ siècle !). On peut rappeler d'ailleurs que cette structure impériale de l'Église date de Grégoire VII qui, dans son Dictatus papae de 1075, proclamait le devoir d'obéissance de tout baptisé au patriarche de Rome, autoproclamé « catholique », pape universel. Cette centralisation pontificale inconnue à ce point jusqu'alors inaugura le Grand Schisme entre Catholicisme et Orthodoxie, les patriarches orientaux ayant refusé ce diktat, parce qu'euxmêmes étaient organisés en système collégial - n'ayant que la primauté morale du primus inter pares $^{23}$.

L'histoire de l'Église catholique est donc un exemple parfait de débordement du religieux sur le politique ainsi que d'une dérive totalitaire de la nature même de celle que l'on dénonce dans les sectes dangereuses... dérive dont la cause fondamentale est le refus des valeurs de la science et de la démocratie, que sont le respect de la majorité et de la critique minoritaire, l'Église catholique étant constituée en monarchie cooptée.

Heureusement aujourd'hui ce n'est plus le cas depuis que la liberté de religion s'est développée à partir de la Révolution française et depuis la loi de 1905 sur la laïcité, l'État s'est séparé de l'Église.

Néanmoins on pourrait encore parler du problème actuel des commandos anti-IVG, condamnés à titre individuel pour les troubles de l'ordre public qu'ils génèrent mais sans donner lieu toutefois à des poursuites contre l'Église, alors que ce fanatisme se nourrit souvent des injonctions mêmes du pape contre l'avortement.

$3^{\circ}$ Islam

Comme le Christianisme à partir de Théodose, l'Islam s'est répandu par la violence de la conquête. Mahomet lui-même de son vivant, après une phase défensive, se lança dans une phase offensive contre les polythéistes arabes qui l'avaient agressé, mais par la suite, la conquête devait envahir de l'Espagne à l'Inde en détruisant temples et universités bouddhistes et hindous ou les transformant en mosquées, en Inde, ou transformant les églises en mosquées, en Espagne.

Aujourd'hui les deux types de violence sont liés à l'Islam : celle de la Charia (a) et celle du terrorisme ${ }^{24}$ (b) interpellent les démocraties afin qu'elles trouvent des parades à la stratégie d'expansion.

a) La violence au quotidien de la Charia

La loi islamique est incompatible avec la démocratie de deux points de vue:

- l'un concerne la souveraineté du droit: en démocratie c'est le peuple qui est souverain, en Islam c'est Dieu (ou plus exactement les Ayatollahs et autres Mollahs, puisqu'on ne connaît de Dieu que ce que les hommes ont bien voulu nous en dire...). Ceci a pour effet:

- un principe de supériorité qui veut que pour les musulmans (sunnites aussi bien que chiites $-95 \%$ des musulmans), la Charia (loi divine - voie, chemin de salut étymologiquement) est forcément supérieure au droit positif et donc au droit démocratique ${ }^{25}$ : le conflit est donc inévitable et insoluble autrement que par le rapport de force;

- des sanctions pénales définitivement fixées. Les sanctions pénales fixées définitivement font que la majorité ne peut modifier les peines (fixées par l'interprétation d'une minorité de Mollahs), situation d'autant plus antidémocratique que les «délits » et les "peines » sont particulièrement flous, n'étant pas précisément définis; aussi l'imprécision de la plupart des sourates concernant ces points permet une interprétation très diverse laissant un maximum de pouvoir à la dictature des Mollahs seulement limitée par les contradictions entre eux! Ainsi, le voleur doit se voir couper la main ${ }^{26}$, la femme adultère doit être lapidée ${ }^{27}$, l'apostate tué (sourate IV, 115).

- L'autre, sur le plan du contenu, choque les règles qui prévalent dans les droits démocratiques :

- la liberté de la vie privée violée par l'immixtion des Mollahs, et en particulier du choix de sa religion et de changer de religion (et le principe de liberté d'association et d'expression qui en découlent), car pour les apostates (ceux qui quittent l'Islam) la peine de mort leur est réservée... Quant à ceux qui combattent l'Islam et ceux qui commettent des violences, il faut les crucifier ou couper leurs membres $(V, 33)$, de même la Charia prévoit par exemple que la femme adultère doit être lapidée et la femme soupçonnée d'infidélité doit être battue ${ }^{28}$;

22. Mais l'Église ne se présente-t-elle pas comme successeur de Pierre, celui qui a renié le Christ au moment difficile?

23. J. Descarreaux, Byzance ou l'autre Rome, Paris, Cerf, 1982, p. 72 et 88.

24. Quand ce n'est pas l'invasion pure et simple (Cachemire, Koweït...).

25. «Telles sont les lois de Dieu : celui qui obéit à Dieu et à son prophète sera introduit dans les jardins [...] ils y trouveront des épouses pures [sourate IV, 56], celui qui désobéit à Dieu et à son prophète et qui transgresse ses lois sera introduit dans le Feu [...] ». Cf. Le Coran, trad. fr. D. Masson, Paris, Gallimard (Pléiade), 1967, IV, 13, 55, p. 95.

26. «Telle sera la rétribution de ceux qui font la guerre contre Dieu et contre son prophète, et de ceux qui exercent la violence sur la terre: il seront tués et crucifiés, ou bien leur main droite et leur pied gauche seront coupés» $(\mathrm{V}, 33)$.

27. «Appelez quatre témoins que vous choisirez contre celles de vos femmes qui ont commis une action infâme. S’ils témoignent: enfermez les coupables jusqu'à ce que la mort les enlève» $(\mathrm{IV}, 15)$.

28. Règles fondées sur l'idée de la sourate IV, 34 qui dispose que «les hommes ont autorité sur les femmes en vertu de la préférence que Dieu leur a accordé sur elles [...]. Admonestez celles dont vous craignez l'infidélité [...] frappez-les [...]». On pourrait également citer les mariages forcés qui du 
- l'égalité entre les citoyens bafouée par les Mollahs, par la discrimination des citoyens dans les pays soumis à la Charia qui doit également être soulignée comme facteur incompatible avec la démocratie: outre le sort peu enviable réservé aux femmes ${ }^{29}$, adultères en particulier (alors que pour l'homme il n'y a pas de sanction), et aux apostates (il n'y a pas de sanction bien sûr pour celui qui se convertit à l'Islam), en effet, les citoyens non musulmans dans les pays où l'Islam est religion d'État sont considérés (et à condition qu'ils soient encore d'une religion issue de la Bible !) comme des citoyens de seconde zone soumis à des discriminations fiscales (IX, 29)... enfin on rappellera aussi que l'Islam accepte l'esclavage comme pratique courante (IV, 3 - captives de guerre -, 36 ; XXIV, 58 ).

Voilà quelques exemples de principes qui, s'ils sont interprétés matériellement (c'est le cas de la plupart des pays musulmans), sont incompatibles avec la démocratie qui laisse la femme libre de sa vie privée bénéficiant de droits égaux à ceux des autres citoyens, et le voleur sanctionné par la prison (sur la base d'une loi modifiable).

\section{b) Le terrorisme islamiste}

Comme on l'a vu la plupart des musulmans, de par cette sourate IV, 13, ne peuvent pas admettre que la loi démocratique prime sur la loi islamique. Deux attitudes sont alors possibles, soit ils ne la respectent pas et risquent « le Feu », soit ils la respectent et vont au « Jardin ». L'incompatibilité est donc manifeste et ne peut que se traduire par un rapport de force qui explique la montée de la violence lorsque les musulmans vivent dans un contexte démocratique, générant une violence latente qui se développe sournoisement par des réseaux clandestins. La seule différence finalement entre les musulmans chiites et sunnites et les islamistes ne réside, on ne peut que le déplorer, que dans le passage à l'acte... et le pas peut être très facilement franchi lorsque la désespérance économique et l'ignorance génèrent la frustration!

La psychologie collective de l'islamisme apparaît également comme une pâle tentative de revanche d'une civilisation qui fut brillante pendant plusieurs siècles mais qui du fait du dogmatisme n'a pas su intégrer les valeurs de la contradiction à partir du $\mathrm{X}^{\mathrm{e}}$ siècle (et définitivement au XIV ${ }^{\mathrm{e}}$ siècle) lorsque la jurisprudence coranique s'est arrêtée, laissant à l'Occident le monopole du développement scientifique et démocratique... et donc de la domination économique, politique et militaire. Cette longue décadence a laissé une énorme frustration, ferment de la violence.

La volonté de destruction de l'Occident et de ses valeurs par les islamistes est la marque même de l'échec de leur idéologie, même si celle-ci attire des marginaux perdants du système occidental qui peuvent alors s'iden- tifier au seul ennemi idéologique de la démocratie qui subsiste après l'effondrement du communisme, l'Islam.

Néanmoins, en attendant, des troubles de l'ordre public sont à attendre et défraient régulièrement l'actualité, obligeant les démocraties à réagir! Les États démocratiques auraient tort de faire la politique de l'autruche comme lors de la montée des totalitarismes fascistes et nazis $^{30}$...

Il semblerait que la Cour européenne des droits de l'homme ait perçu le danger à travers un arrêt du 31 juillet 2001 rendu sur un recours rendu par la Cour constitutionnelle de Turquie qui avait entériné la dissolution d'un parti islamiste ( de la Prospérité») risquant de prendre le pouvoir ( $22 \%$ des voix aux élections législatives de 1995).

La Cour européenne n'a pas sanctionné la Turquie aux motifs que la dissolution était « légale», c'est-à-dire prévue par une loi (au sens large) et non contraire aux nécessités d'un État démocratique. En effet l'article 11 de la Convention justifie la légitimité des atteintes aux libertés dès lors qu'elles visent la sécurité nationale, la défense de l'ordre ou la protection des droits et libertés d'autrui et à condition que les atteintes soient proportionnées au but légitime suivi. Dans l'espèce la Cour a admis l'exception prévue par la Convention, car le dirigeant du parti en question avait tenu des propos quelque peu alarmants sur son programme politique axé sur trois aspects : l'instauration de la Charia, du Djihad, et d'un système multijuridique (la Charia applicable aux musulmans). En effet dans un discours de mars 1993 Necmettin Erbakan a soutenu l'idée qu'il « devait y avoir plusieurs systèmes juridiques puisqu'il y avait plusieurs courants religieux» en Turquie. En 1994 et 1996 le même président affirmait que «l'ordre juste serait établi et qu'il faut féliciter ceux qui contribuent... à la suprématie d'Allah ». Entre 1993 et 1997 un député de ce parti avait affirmé : « Je veux instaurer la Charia. Le passage [à l'ordre juste] sera-t-il pacifique ou violent, se fera-t-il en douceur ou dans le sang? Les 60 millions [de citoyens] doivent prendre position sur ce point $[\ldots]$. Je voudrais que le sang coule. C'est ainsi qu'arrivera la démocratie. Et ce sera bien beau. » Cet arrêt a été diversement accueilli et soulève de nombreuses questions, dont une, essentielle, a été soulevée par le Doyen Gilles Lebreton dans un article remarqué ${ }^{31}$, à savoir: une Cour européenne peut-elle au nom de la protection de l'État de droit s'opposer à la volonté du peuple qui semblait vouloir élire démocratiquement ce parti (et qui l'a fait depuis, le parti dissout s'étant reformé sous un autre nom...) ? Ne risque-t-on pas de glisser vers le gouvernement des juges ${ }^{32}$ qui serait une récupération du pouvoir au profit de quelques-uns (dont on rappellera qu'ils n'ont aucune légitimité démocratique, n'étant

point de vue de notre droit constituent un viol de mineures, ou encore la pratique de l'excision (qui sont des crimes sur le plan pénal en France, mais dont il faut noter que si cette pratique est traditionnelle dans certains pays musulmans, ce n'est pas un précepte coranique).

29. Dont l'héritage est la moitié de celui d'un garçon (IV, 11).

30. Deux ouvrages en particulier dénoncent ce danger : celui d’A. Del Valle, Le Totalitarisme islamique à l'assaut des démocraties (Paris, Éditions de Syrtes, 2002) et celui d'I. Warraq, Pourquoi je ne suis pas musulman, Lausanne, L'Âge d'homme, 1999.

31. Dans la «Chronique européenne » de la Revue de droit public, $\mathrm{n}^{\circ}{ }_{5}, 2002$.

32. Les juges européens, par un ton condescendant particulièrement choquant, évoquent le fait que «la démocratie suppose de donner un rôle au peuple». 
pas élus!), négation même de la démocratie? La question est, on le voit, particulièrement brûlante.

Le Doyen Lebreton fait une critique incontestable: le fondement juridique de la décision européenne est clairement abusif eu égard à l'absence de menace concrète et immédiate sur les libertés du peuple turc. La liberté d'expression devant être absolue, même si elle choque, devait être garantie par la Cour et ne saurait aboutir à la dissolution d'un parti !

En effet le danger, dans la politique intérieure, est de reprendre les méthodes fascistes de répression même préventive, car alors la démocratie se dévoie elle-même (on connaît le danger de la formule célèbre de Saint-Just au moment de la Révolution: " pas de liberté pour les ennemis de la liberté»). Néanmoins le Doyen Lebreton fait deux autres critiques que nous ne suivrons pas, car si sur le plan théorique elles sont pertinentes, sur le plan stratégique et réaliste, elles semblent trop optimistes. Ainsi il oppose la philosophie et l'humanisme à cette décision.

Sur le premier point il a raison de souligner le danger pour les démocraties de recourir elles-mêmes aux méthodes qu'elles combattent, et nous sommes pleinement en accord avec cette idée.

Sur le plan de l'humanisme, il souligne que la Charia et l'Islam vus de cette façon fondamentaliste sont caricaturés. Il oppose en effet la critique linguistique et historique des versets coraniques et de la Sunna (la tradition des haddith) à la rigidité des injonctions appliquées en droit positif. En particulier il souligne justement que les règles coraniques visaient à améliorer le droit antérieur en fixant des règles plus évoluées (vendetta remplacée par la loi du talion ${ }^{33}$, etc.), mais qu'aujourd'hui ces règles seraient obsolètes.

Pourtant deux arguments s'opposent à cet optimisme :

- la critique historique est rejetée par le clergé musulman (comme elle le fut en son temps par le clergé chrétien ${ }^{34}$ ), car de quelle autorité un juriste ou un historien déciderait que telle loi divine serait ou ne serait plus applicable, obsolète? De quelle autorité l'homme déciderait de supprimer une loi imposée par Dieu? Il y a là une incompatibilité majeure vis-à-vis du point de vue dogmatique. La seule possibilité d'attaquer sur ce point est celle de la traduction et de l'interprétation, car alors on ne touche pas au corpus en faisant une sélection (donc on ne touche pas au caractère «parfait» et «sacré» du texte en soi), on conteste simplement la réalité même de la règle prétendument déduite du texte sacré par l'interprétation faite par le clergé depuis les califes, qui ont sans doute eux-mêmes poussé dans le sens matériel afin d'asseoir leur pouvoir temporel. En outre les concepts pénaux coraniques étant rarement définis on peut contester aux Mollahs le monopole de les interpréter, en particulier de façon exclusive et sociale (les nombreuses divergences en sont la preuve), et au contraire on peut envoyer chacun à l'interprétation du texte, dans le cadre de sa vie privée. On soulignera précisément que la seule interprétation compatible avec la démocratie et l'humanisme est celle des soufis, qui renvoie le Coran à la pratique spirituelle personnelle. En effet dans cette interprétation, la Charia est le chemin intérieur vers Dieu, et le Djihad la guerre intérieure contre l'ego : ainsi c'est le voleur en moi (avidité, jalousie) à qui il faut que je coupe la main ; c'est la femme adultère en moi (esprit volage) que je dois lapider. Cette interprétation du Coran permet alors de reléguer les préceptes religieux dans la sphère de la vie privée, au niveau de la recherche et de la pratique spirituelle personnelle. Mais le soufisme ne représente aujourd'hui qu'une infime partie de l'Islam (peut être $2 \%$ ).

- La réalité sociologique et historique montre qu'aujourd'hui la quasi-totalité des pays musulmans applique la Charia matérielle, rigide ${ }^{35}$, avec les règles précitées, depuis plus 1000 ans; il serait donc complètement irréaliste, voire dangereux, de voir l'Islam comme un humanisme, vus les faits. Si les démocraties (et l'État français en particulier qui a refusé longtemps la reconnaissance des structures musulmanes à cause de cette ambivalence, et qui vient malheureusement d'y mettre fin sans discernement) voulaient être cohérentes et claires, elles accepteraient de reconnaître les structures soufies, compatibles avec la démocratie et ne devraient pas donner la personnalité morale aux autres pour incompatibilité avec l'ordre public (la dissolution est même prévue par la loi de 1901 en France)! Cela ne serait pas une atteinte à la liberté d'association (qui resterait possible en créant des associations de fait) mais en n'ayant pas de personnalité morale, elle ne pourrait pas avoir une puissance concrète de diffusion et d'extension. Pourquoi la démocratie reconnaîtrait-elle des structures qui la combattent férocement? Pour autant on ne saurait sanctionner des propos fondamentalistes dans une démocratie, au risque de porter atteinte à un droit fondamental de contestation du système, le débat d'idées quel qu'il soit doit être protégé absolument et sans aucune restriction.

\section{b. Le fanatisme dans les religions védiques $1^{\circ}$ Bouddhisme}

On ne connaît pas d'exemple historique où le Bouddhisme aurait été à l'origine de violence physique ou de guerres de conquête. C'est la seule religion qui se soit vraiment développée librement et sans violence.

On pourrait rétorquer que la période féodale du Tibet a connu pas mal de violences et a abouti à l'intronisation des dalaï-lamas. Mais cette institution politique n'a rien à voir avec le Bouddhisme. En effet les dalaï-lamas, chefs de l'École Guelougpa, ont été mis sur le trône par le fils de Gengis Khan qui était disciple du maître de l'époque. Mais cette structure qui n'a rien de bouddhiste est même

33. Elle-même datant du code d'Hammourabi (XVIII' siècle av. J.-C. environ).

34. Une des causes essentielles de la croisade anticathare était qu'ils avaient été les premiers à traduire les textes sacrés et leur avaient donné une interprétation différente de l'Église.

35. On constate même un retour en arrière et une tendance au développement du fondamentalisme dans certains pays musulmans, comme au Nigéria avec des femmes condamnées à la lapidation pour adultère. 
contraire aux préceptes du Bouddha qui dans le Vinaya Pittaka enseignait de ne pas mettre de chef à la tête de ses communautés mais simplement un "porte-parole» élu ad hoc ${ }^{36}$ pour une négociation extérieure et sur la base d'une décision prise consensuellement (c'est-à-dire jusqu'au silence) par les moines ${ }^{37}$. S'il en est résulté des tensions entre les quatre écoles tibétaines, c'est sans rapport avec la doctrine du Bouddha.

Aujourd'hui le Bouddhisme est sans doute la seule religion effectivement non violente et adaptée à la modernité démocratique et scientifique, le Bouddha ne disait-il pas: «Si votre expérience diffère de ce que je dis, croyez votre expérience et pas ce que je dis »...?

Les conflits entre le Bouddhisme, le Taoïsme et le Confucianisme chinois, le Shinto au Japon, la religion Bön au Tibet, son restés du point de vue bouddhiste des conflits de doctrine, et s'il y a pu y avoir des rapports de force, c'était lorsqu'il y avait des enjeux de pouvoir : unifier un territoire, asseoir une autorité...

\section{$2^{\circ}$ Hindouisme}

De son coté l'Hindouisme a été peu enclin aux violences également, mais néanmoins plus que le Bouddhisme. En effet le système de castes, bien que système d'ordre, est devenu avec la décadence du Brahmanisme hiérarchisé et héréditaire ${ }^{38}$. Ceci a figé l'ordre social dans l'injustice et l'inefficacité, et la faiblesse de la société qui en a résulté a facilité les invasions musulmanes puis européennes, grâce auxquelles les basses castes se sont converties à la religion de l'envahisseur, échappant à leur vieux carcan et leur donnant l'occasion de prendre leur revanche sociale, mais c'est l'agressivité et le triomphalisme des musulmans (destruction des temples hindous et construction des mosquées dessus) qui ont provoqué des réactions violentes, qui se maintiennent aujourd'hui dans les conflits comme ceux du Cachemire.

Les rares problèmes entre hindous et bouddhistes ${ }^{39}$, à Ceylan, ne sont en réalité qu'un problème politique hérité des Britanniques, passés maîtres dans l'art de diviser (prétextant les religions) pour mieux régner: ils avaient en effet introduit une minorité hindoue à Ceylan pour gouverner la région (gouvernement autoritaire et corrompu) qui a été balayée lors de l'indépendance et l'instauration de la démocratie, mais la minorité étant riche et puissante le conflit ne pouvait qu'éclater. Cependant il s'agit d'un conflit ethnico-politique et non religieux. Par ailleurs les religions traditionnelles sont aussi facteur de paix sociale.

\section{La paisibilité dans les religions traditionnelles}

Quand la vie religieuse est cantonnée à la vie privée il n'y a pas de violence. Chacun vit sa croyance et sa pratique, dirige ses actes selon sa propre morale personnelle.

Toutes les grandes religions dans leur dimension spirituelle respectent les autres, et s'opposent à la violence et à la volonté de puissance, liées à la loi de la jungle, loi animale du plus fort, lui substituant la loi d'amour et de compassion.

Quand elles sont tournées vers l'intérieur et quand elles s'occupent des autres pour aider ceux qui souffrent alors les religions sont des facteurs de la paix sociale: tous les monastères du monde sont des lieux de paix (intérieure certes, mais aussi extérieure, ce qui importe en démocratie). Si la violence peut y exister à l'intérieur (les conflits humais existent partout !), c'est alors l'objet du travail sur la maîtrise de soi que tentent de réaliser les moines. De plus les grandes religions ont également généré beaucoup de créativité artistique, scientifique, philosophique, qui ont servi de terreau à la modernité. C'est vrai pour toutes les religions : Taoïsme, Bouddhisme, Hindouisme, Judaïsme, Chritianisme, Islam soufi.

Les nouvelles religions feraient-elles exception ou suivent-elles un autre schéma?

\section{B. Les nouvelles religions et la laïcité}

Pour ce qui est des nouvelles religions comme pour des anciennes (mais avec beaucoup moins de morts néanmoins... ${ }^{40}$ ) on trouve des mouvements dangereux pour lesquels le terme secte est approprié quand des actes illégaux sont commis en rapport avec une doctrine (1), et d'autres au contraire n'ont pas eu de condamnation (2).

\section{Les nouvelles religions sectaires}

Quelques groupes ont défrayé la chronique avec des morts ou des actes manifestement illégaux :

- le Temple du Peuple: suicide et assassinats : 914 morts en 1978 à Jonestown;

- les Davidiens: suicide (ou exécution du FBI?) à Waco (Texas) en 1986, quelques dizaines de morts;

- OTS : suicide et / ou exécution (l'instruction a été contestée) : quelques dizaines de morts en 1994 et 1997;

- le groupe californien Heaven's Gate s'est suicidé en 1997 lors du passage d'une comète: quelques dizaines de morts...;

- le groupe Shinrikyo (Aum) avec ses attentats au gaz à Tokyo en 1995 a fait 12 morts... ;

- la Famille, dont certains membres ont été condamnés pour des affaires sexuelles.

Sur les 172 mouvements qualifiés de sectes en France, il n'y a guère qu'un ou deux groupes criminels réellement implantés dans notre pays (l'OTS) : c'est bien peu! Et

36. Cf. M. Wijayaratna, Le Moine bouddhiste, Paris, Cerf, 1983, p. 165.

37. Cf. Le Bouddha historique de l'historien Hans Wolfgang Schumann (Vannes, Sully, 1999).

38. Ce qu'il n'était pas encore à l'époque des grandes épopées comme le Ramayana, car on y présente l'exemple d'un Ksatrya - caste guerrière - devenant Brahman - caste des prêtres.

39. Il est à noter que contrairement au Christianisme qui a été rejeté comme hérétique par les juifs, les brahmanes ont reconnu le Bouddha comme le $9^{\mathrm{e}}$ avatar de Visnu (principe cosmique protecteur), donc l'Hindouisme en quelque sorte inclut le Bouddhisme (mais ce n'est bien sûr pas le point de vue des bouddhistes!).

40. Les morts du fait des «sectes» se comptent en quelques centaines (Guyana, Temple Solaire, etc.), même si c'est toujours trop, il n'y a aucune commune mesure avec les morts issus des conflits des religions traditionnelles! 
pourtant quel battage médiatique par rapport aux dégâts des religions traditionnelles. Les médias avaient d'ailleurs prédit au tournant du millénaire que les sectes apocalyptiques s'autodétruiraient... 2000 et 2001 sont passés sans le moindre suicide! Mais le plus grave est l'amalgame volontairement créé par certains politiques (rapports parlementaires de 1995 et 1999) et entretenu régulièrement par des médias en mal d'audimat. En utilisant quelques affaires pénales marginales, les ennemis de la liberté de religion y associent par amalgame des mouvements qui n'ont aucun rapport et créent ainsi des bouc-émissaires. Il s'agit d'une tactique très ancienne utilisée déjà par les Romains qui l'utilisaient contre les chrétiens dans l'Antiquité.

\section{Les nouvelles religions au sectarisme contesté}

Quels sont les groupes condamnés pour des actes illégaux en rapport avec leur doctrine, si l'on admet cette définition qui est la seule à être cohérente et compatible avec le principe démocratique?

- La Scientologie s'est vue condamnée par un procès à Lyon pour escroquerie ${ }^{41}$. Néanmoins il n'a pas été démontré que l'escroquerie était liée au fonctionnement de la Scientologie, seul un "déviant» a été condamné (interdirait-on l'Église catholique à cause de la condamnation d'un prête pédophile?);

- le Mandarom a été condamné pour sa statue alors qu'il s'agit d'une incohérence administrative qui a donné un permis tacite refusé pourtant par la Cour de cassation $^{42}$ sur des motifs fallacieux et contradictoires. Mais là encore, cette condamnation douteuse est de toutes façons sans rapport avec sa doctrine qui prône au contraire la synthèse des religions et la paix entre les êtres!

- IVI qui pratique des prières pour soulager la souffrance est considérée comme faisant de la médecine illégale. Deux médecins de ce mouvement ont subi des sanctions disciplinaires de l'ordre des médecins (là encore condamnations discutables mais qui de toutes façons n'engagent pas le mouvement car ce sont des pratiques personnelles de certains médecins). En outre pourquoi IVI est il reconnu d'utilité publique en Allemagne? L'Allemagne ne serait-elle pas un pays démocratique et civilisé ?

- Horus qui fait du bio-ésotérique, condamné pour non-assistance à personne en danger et médecine illégale par rapport à une adepte cardiaque et très âgée décédée dans ses locaux (là encore la justice n'a pas montré beaucoup d'objectivité);

- les Témoins de Jéhovah, sur le conflit du sang, ont finalement obtenu gain de cause, du fait du scandale du sang contaminé...

\section{Les autres mouvements ?}

Sri Chinmoy avec sa course pour la paix et sa médiation devant l'icône de son fondateur n'a jamais été con- damné; la Méditation transcendantale également n'a jamais été condamnée..., ni les autres parmi les $172 \ldots$ et la liste est longue!

Certains parmi ces mouvements ne sont le plus souvent que poursuivis que par des administrations, bras armés des politiciens au pouvoir : fisc, URSSAF, ce qui en dit long sur l'objectivité de ces poursuites!

Le monde associatif étant un monde d'une grande liberté de structure (la loi de 1901 est particulièrement elliptique, contrairement à la loi sur les sociétés commerciales) où les fondateurs ne sont pas des juristes, il est facile de constater des irrégularités sur le plan fiscal et social. Mais si toutes les associations exerçant en France étaient vérifiées il n'en resterait plus beaucoup ! Le choix d'attaquer certaines est donc un choix purement politique ou religieux, voire discriminatoire.

D'autre part la plupart de ces mouvements sont pacifiques et pour beaucoup prônent également la paix sociale dans leur doctrine (Méditation transcendantale, Mandarom, etc.).

\section{Plaidoyer pour une investigation sérieuse sur les mouvements religieux}

Après les rapports parlementaires partisans, rédigés sans la moindre consultation de laboratoires universitaires, sur la base de concepts flous, non définis et contestables, portes ouvertes à toutes les dérives tendancieuses, sans aucun contradictoire, il apparaît indispensable de repenser la recherche sur le plan de la religion. L'étude de méthodes d'investigation des mouvements religieux nécessite que l'on envisage tout d'abord des points de repères qui sont ceux qui prévalent dans nos sociétés modernes occidentales afin de servir de fondement à une méthode d'investigation crédible (A) avant d'en déduire plus concrètement les étapes de la méthode de travail que doit adopter un Observatoire des mouvements religieux (traditionnels ou non) (B).

\section{A. Le fondement conceptuel de la méthode de travail d'un Observatoire objectif}

Notre société moderne est fondée sur deux critères qui sont nés sur le sol européen comme on l'a vu plus haut, à savoir : la démocratie et la démarche scientifique.

On a également constaté d'ailleurs qu'en matière de religion (et de secte en particulier), c'est la plupart du temps les mécanismes inverses que l'on constate: une minorité ecclésiastique impose son interprétation des textes, et celle-ci est dogmatique, c'est-à-dire que l'on ne peut la remettre en question.

41. La Cour d'appel de Lyon dans un arrêt du 28 juillet 1997 a réaffirmé le principe de laïcité: «Attendu qu'il serait vain dès lors de s'interroger sur le point de savoir si l'Église de scientologie constitue une secte ou une religion, la liberté de croyance étant absolue; que dans la mesure où une religion peut se définir par la coïncidence de deux éléments, un élément objectif, l'existence d'une communauté même réduite, et un élément subjectif, une foi commune, l'Église de scientologie peut revendiquer le titre de religion.»

42. Arrêt de la Chambre criminelle du 19 septembre 2000. 
Cette opposition radicale constitue probablement la raison pour laquelle aujourd'hui les sociétés démocratiques et scientifiques se heurtent aux obscurantismes et aux fanatismes de tous bords qui tentent de s'opposer à son extension: sur le plan externe on peut constater en effet que la plupart des guerres aujourd'hui sont des guerres de religion (Irlande, Israël, ex-Yougoslavie, Soudan, Tibet...), et sur le plan interne on constate une recrudescence des fanatismes identitaires, qui tentent de s'opposer au métissage humain et culturel lié aux échanges internationaux. Cette recherche d'identité se manifeste par un renouveau des intégrismes, mais aussi par l'émergence de nouveaux mouvements religieux, dont certains, socialement pathogènes, utilisent le paravent religieux pour dissimuler leur volonté de puissance, ce ne sont plus alors des mouvement religieux authentiques.

Mais ces nouveaux mouvements présentent une critique de notre système, et en cela notre société doit les prendre en considération et les respecter ${ }^{43}$, mais à condition qu'eux-mêmes respectent la légalité majoritaire!

On peut donc diviser les mouvements religieux en deux catégories : d'une part ceux qui respectent la légalité, d'autre part ceux qui ne la respectent pas.

Les premiers sont tout à fait respectables et doivent bénéficier de la protection républicaine au même titre que les religions anciennes; les seconds sont les sectes ${ }^{44}$ qui doivent être dénoncées, poursuivies et dissoutes.

Dans un État de droit il ne peut qu'en être ainsi: seule l'illégalité des comportements peut être poursuivie et sanctionnée ${ }^{45}$. Il est donc capital d'étudier les mouvements religieux en détail pour constater leur éventuel caractère sectaire, afin d'éviter tout amalgame, qui consisterait à dire (et plus généralement à penser) : «tout ce qui n'est pas ma religion est une secte...» C'est précisément l'objet d'un Observatoire objectif. Quelles peuvent être les méthodes de travail utilisées?

\section{B. Le fonctionnement d'un Observatoire}

L'exigence démocratique et scientifique se résume en deux concepts : esprit critique et consensus. Pour cela on doit envisager la méthode de travail d'une part par rapport aux enquêteurs de l'Observatoire, sa composition (1), puis les étapes de la méthode elle-même (2).

\section{La composition de l'Observatoire}

Plusieurs règles doivent être réunies afin de garantir la qualité des travaux d'un tel Observatoire. La première concerne l'appartenance (a), la seconde, la compétence scientifique (b), puis d'autres règles de fonctionnement quant à la désignation des rapporteurs (c), ainsi que d'élaboration des rapports d'enquête (d).

a) Afin d'assurer l'objectivité des travaux, une règle statutaire doit imposer à un Observatoire qu'aucun de ses membres n'appartienne à un mouvement religieux (ou politique lié à une religion). En effet, s'il n'est pas possible d'empêcher quiconque d'avoir ses sympathies (ni même de les connaître dans leur réalité!), il est en revanche d'un minimum d'objectivité que de ne pas appartenir à un mouvement pour ne pas être juge et partie $^{46}$ !

b) L'autre garantie d'objectivité et de rigueur réside dans le fait que ses membres doivent tous avoir une formation universitaire validée, qu'ils soient enseignantschercheurs ou praticiens, afin que l'analyse des mouvements soit conforme aux protocoles scientifiques, ce qui n'était pas le cas de l'aveu même des rapports de 1995 et 1999. Pour un Observatoire de juristes, comme des praticiens avocats peuvent être utiles de par leur expérience, et qu'ils doivent avoir au minimum une maîtrise en droit, un tel niveau devrait être nécessaire au minimum pour appartenir à l'Observatoire.

On pourrait considérer a priori que la présence d'avocats (dans un Observatoire de juristes) qui dans leur vie professionnelle ont eu l'occasion de défendre certains mouvements religieux ou des victimes de sectes risque d'influencer les analyses.

Mais on peut répondre que l'objectivité de l'Observatoire ne devrait pas être nécessairement affectée pour autant et ce, d'une part pour une raison essentielle relative à la déontologie de l'ordre des avocats ${ }^{47}$, car ce n'est pas parce qu'un avocat défend un assassin qu'il est luimême complice de l'assassin! D'autre part on peut et on doit veiller à ce qu'un avocat qui a été défenseur d'une partie dans un procès avec un mouvement religieux (défenseur d'une victime ou d'un mouvement) ${ }^{48}$, ne soit pas rapporteur ou enquêteur de ce mouvement.

Les autres garanties se retrouvent dans les règles suivantes :

43. Ils font apparaître une insatisfaction fondamentale, qui est la marque d'une insuffisance de ce système, alors que le but (idéal) du droit est de satisfaire tous les citoyens sur tous les plans. Ces critiques doivent être un stimulant pour faire évoluer ce système (que ce soit au niveau de l'État - Éducation nationale qui refusa longtemps d'enseigner l'histoire des religions, créant des générations d'acculturés, proies faciles pour les sectes - ou de l'éducation religieuse traditionnelle et familiale défaillante - qui fait fuir les jeunes dans les sectes).

44. On donnera donc de la secte la définition suivante: Est une secte, tout mouvement religieux dont la doctrine incite les adeptes à commettre des actes illégaux en rapport avec sa doctrine.

45. Ce qui rend tout à fait contradictoire (et en tous cas incompatible avec le principe de la légalité des peines prévu par l'article 8 de la Déclaration des droits de l'homme de 1789) la position du Rapport Guyard Gest de 1995 selon laquelle pour qu'il y ait condamnation d'un mouvement «il faut aussi que les faits correspondent à une incrimination prévue et sanctionnée par la loi, ce qui n'est pas évident dans les cas de manipulation mentale par exemple» (2.B.2).

46. Contrairement aux groupes de pression antisectes, utilisés comme bases de données par les rapporteurs de l'Assemblée nationale, comme l'Adfi dont un des fondateurs est un prêtre catholique (le père Trouslard) qui se prend pour un nouvel inquisiteur...

47. On entend çà et là (Figaro du 28 avril 1996) que l'ordre des médecins doit être associé à un Observatoire public. L'idée est intéressante (bien qu'elle n'apparaisse pas confirmée par le décret précité), mais pourquoi ne pas y adjoindre alors l'ordre des avocats et le Conseil national de la magistrature, voire d'autres académies ou laboratoires de recherches universitaires?

48. La présence des deux variétés serait souhaitable afin de garantir le contradictoire et la transparence des débats. 
c) afin d'éviter toute tentative d'infiltration, l'affectation des rapporteurs aux mouvements à étudier devrait se faire par tirage au sort (après vérification des points cidessus) ; le rapport préalable d'enquête devant être signé du rapporteur qui l'aura réalisé afin de toujours pouvoir identifier l'origine d'une enquête ;

d) enfin tous les rapports à publier devraient être élaborés par l'ensemble de l'Observatoire à la majorité, reflétant donc le consensus des spécialistes dans leur ensemble et non l'opinion d'un membre ou d'un autre en particulier...

Après avoir vu les meilleures conditions d'objectivité par rapport aux membres de l'Observatoire, on peut aborder maintenant l'élaboration des rapports sur les mouvements religieux.

\section{Les étapes de la méthode d'investigation}

On n'envisagera ici que la méthode d'un Observatoire juridique ${ }^{49}$, chaque science ayant ses propres protocoles.

Deux écueils sont à éviter dans cette démarche : d'une part être piégés par les mouvements, leurs discours dogmatiques et leur langue de bois; d'autre part être piégés par les opposants à tel ou tel mouvement et dont le point de vue est généralement tout aussi partial.

Mais si l'esprit critique à l'égard des deux est bien sûr de rigueur, il faut néanmoins utiliser des documents qui en émanent (a) afin de faire apparaître des contradictions significatives qui pourraient naître de la vérification point par point de toutes les informations alléguées (b), permettant ensuite d'en faire la synthèse (c).

\section{a. Première étape (thèse)}

Dans une première étape, il serait utile d'envoyer un questionnaire d'une centaine de questions à remplir par les dirigeants des mouvement religieux, relatives à trois domaines:

1) bien sûr les aspects juridiques au premier chef:

- les aspects juridiques internes quant au fonctionnement du mouvement, les hiérarchies... pour déceler ceux qui sont illégaux, en priorité, mais aussi ceux, qui, sans être illégaux en soi, peuvent induire un dérapage vers l'illégalité (ainsi par exemple les fonctionnements non démocratiques peuvent-ils être pathogènes - abus de pouvoir etc. -, bien que pas interdits par la loi du $1^{\text {er }}$ juillet 1901);

- mais surtout les aspects juridiques externes par l'étude des décisions de justice ${ }^{50}$ qui auront été rendues à leur encontre.

Pour cela il est clair qu'il y a besoin des informations émanant des mouvements eux-mêmes (d'où le questionnaire). Cette étude est nécessaire car elle permettrait de faire apparaître la spécificité des contentieux liés au mouvement à étudier. En effet par exemple un redressement fiscal n'est pas spécifique aux sectes, sinon, toutes les personnes morales redressées seraient des sectes... hypothèse absurde ${ }^{51}$. De même il faut faire un tri entre les décisions personnelles et celles qui touchent la doctrine criminogène, seule à nous intéresser ici : en effet un viol opéré par un membre de secte à titre personnel ne nous intéressera pas, en revanche s'il est lié à la doctrine - ou à la pratique du mouvement - il est significatif).

Indépendamment des aspects juridiques purs, d'autres aspects peuvent intéresser les juristes dans ce questionnaire :

2) d'une part la dimension psychologique, verticale, spirituelle enseignée par le mouvement;

3) mais aussi la dimension dogmatique de la doctrine.

En effet, ce sont tous deux des éléments qui peuvent donner des indications précieuses sur la dangerosité potentielle des mouvements étudiés (en particulier la pression sur le consentement, les violences, les vols). La coopération avec les spécialistes de ces disciplines est donc essentielle.

\section{b. Seconde étape (vérification critique / antithèse)}

Cette étape est capitale car elle va être constituée de la vérification de toutes les informations fournies par le questionnaire :

1) d'une part en étudiant les textes et les enseignements émanant de ces mouvements, afin de vérifier que ce qui a été répondu dans le questionnaire correspond bien aux enseignements eux-mêmes;

2) d'autre part en étudiant les jurisprudences concernant les mouvements étudiés, en les comparant au

49. La logique de la science juridique inclut elle-même la logique formelle de type inductif ou déductif et la logique rhétorique (cf. J. Ghestin et G. Goubeaux, Traité de droit civil, Paris, LGDJ, 1983, t. I, p. 28 sq.) ; ainsi comme toute science, la science juridique, en se fondant sur le principe d'identité, utilisera le processus inductif pour fonder l'hypothèse (équivalence générale), la méthode déductive pour la démonstration de l'hypothèse (par des équivalences intermédiaires) et enfin le processus probabiliste pour sa vérification (équivalences externes) (cf. P. Gast, Expérience spirituelle, conscience et droit, Paris, Librairie de l'Inde, 1993, p. 24 sq.), l'ensemble de ces processus pouvant se regrouper sous le terme de thèse. Laquelle doit pour être solide résister aux antithèses afin d'être présentée dans une synthèse. L'ensemble pouvant être seulement alors, qualifié de dialectique. On remarquera que l'idée essentielle de cette méthode reste le principe d'identité, fondement de toute recherche scientifique qui a pour corollaire la vérification systématique d'une information (ce qui fait malheureusement le plus défaut dans le Rapport Guyard Gest et dans les lynchages médiatiques - mais il est vrai que l'audimat et la rigueur font rarement bon ménage...).

50. S'il est possible de prendre en compte les décisions pour lesquelles un recours est encore possible, ce ne doit être qu'avec la plus grande prudence car seule une décision sans recours possible peut être considérée comme définitive, ayant seulement alors l'autorité de chose jugée, seule certitude en matière juridique.

51. Ce point en particulier apparaît comme une faiblesse essentielle dans la démarche du Rapport Guyard Gest de 1995: outre le flou des dix critères retenus, il cite comme on l'a vu, en particulier les « démêlés judiciaires» et le « détournement des circuits économiques» (1.B.1 et 3.A.1.b) affirmant également que "Sur ces bases, ont été recensées [...] les associations remplissant l'un au moins de ces dix critères» (1.B.1). Ainsi par exemple, n’importe quelle personne morale qui aurait des « démêlés judiciaires » devrait être qualifiée de secte (les partis politiques?)! Prenons un exemple : celui des conflits fiscaux (alliant les deux critères précités). N'est-on pas en plein dans l'absurde si l'on doit considérer que toutes les personnes morales qui ont eu un redressement fiscal sont des sectes? Et pourtant c'est ce qui a été fait par ce rapport pour 172 mouvements - sans pour autant d'ailleurs apporter la preuve pour chacun d'eux, d'au moins un seul de ces critères! Comme on le constate on est en plein dans l'arbitraire... 
questionnaire pour voir si un oubli ne serait pas l'indication d'une éventuelle dissimulation d'informations (informations qui, elles, pourraient être fournies éventuellement par des associations antisectes);

3) et enfin par des enquêtes sur le terrain (afin de vérifier que les dires correspondent à la pratique), opérées par des groupes d'au moins trois spécialistes (tirés au sort parmi les membres de l'Observatoire, pour éviter toute pression et limitant les risques de partialité) qui collecteraient des informations : par interview des adeptes actuels, mais aussi d'anciens adeptes, afin de garantir toujours le contradictoire; témoignages signés de leurs auteurs (comme pour le questionnaire) afin d'éviter toute interprétation personnelle des enquêteurs.

En outre les difficultés rencontrées (en particulier le refus de donner une information demandée) devraient être consignées dans le rapport, ainsi que toutes les contradictions qui apparaîtraient entre le questionnaire, les textes, les jurisprudences, les enquêtes et les témoignages.

\section{c. Troisième étape (synthèse)}

Elle devrait être constituée par l'élaboration d'un rapport de synthèse destiné à la publication, qui devrait être élaboré selon le plan ci-dessus, sur la base du rapport préliminaire d'enquête, par les membres de l'Observatoire réunis en assemblée, de façon dialectique (critique permettant de palier d'éventuelles insuffisances des enquêtes préliminaires) et consensuelle (vote à bulletin secret sur chaque point critiqué ou amendé).

\section{d. Conclusion}

Cette procédure, et en particulier le questionnaire, doit être un point de départ que l'on devrait pouvoir adapter en fonction de son efficacité.
Ainsi il parait nécessaire qu'il y ait plusieurs phases d'enquêtes, chacune amenant une précision plus grande dans l'analyse des mouvements étudiés ${ }^{52}$, ne serait-ce que parce qu'un mouvement peut évoluer en fonction des rapports et finir par se conformer à la légalité républicaine.

Telle semble être la meilleure méthode d'approche des mouvements religieux, sectaires ou non, celle qui amènerait une véritable analyse non discriminatoire et crédible du phénomène, seule à même de permettre de trouver des mesures préventives efficaces, protégeant à la fois les personnes fragiles effectivement, mais aussi la liberté des mouvements religieux authentiques.

\section{Conclusion générale}

Tant que le problème de la mort ne sera pas résolu, il y aura toujours de nouvelles théories sur l'immortalité et de nouvelles techniques pour y accéder comme c'est le cas depuis des dizaines de milliers d'années. Tenter de s'y opposer est ridicule, contraire à la nature et à la définition même de l'être humain qui avant même d'être sapiens est, et restera homo religiosus en quête de son identité profonde. La laïcité doit au moins être neutre, et si possible favoriser la diversité des hypothèses et pourquoi pas intégrer le mécanisme démocratique et scientifique dans la spiritualité et inversement: à quand une science de la spiritualité ? Comme le pensait Voltaire en substance, plus il y a de religions, plus il y a de liberté de religion... Sur le plan concret et juridique, il faut que toutes les religions, anciennes et nouvelles, respectent les principes de la laïcité, et qu'en échange la République leur reconnaisse la personnalité morale sans discrimination et sans favoritisme (et pour cela une rationalisation de la loi de 1905 serait la bienvenue!). 
